\title{
Enhancing simulation in complex systems
}

\author{
R. M. Alqirem \\ Department of Management Information Systems, \\ Al-Zaytoonah University of Jordan, Jordan
}

\begin{abstract}
Simulation is an important tool in understanding and designing physical systems, engineering systems and social systems. Because of its importance and broad range, it has been the subject of numerous research studies and books. Simulation is about techniques for using computers to imitate (simulate) the operations of various kinds of real world complex systems. It has been an accepted tool for the improvement of decision making through learning how to deal with the complexity of the real world. The complexity slows the learning loop and reduces the learning gained on each cycle.

This paper illustrates the importance of system thinking in enhancing the simulation process and providing the ability to see the world as a complex system, where "you cannot just do one thing" and that "everything is connected to everything else". It is a holistic worldview that enables people to act in consonance with the best interests of the system as a whole and thus enhance the learning loop through various system thinking tools. The case study in this paper illustrated the use of a system dynamics simulator to allow the financial manager in a firm to test different account receivables scenarios and the strategies to control these accounts. We found that this simulator has helped the manager to get a deeper insight into the effect of their decisions and the different interrelated variables that involve with setting a strategy to control account receivables.
\end{abstract}

Keywords: systems thinking, complex systems, systems dynamics, simulation.

\section{Introduction}

As the world becomes more complex, many people and organisations find themselves bombarded with lots of problems to solve, less time to solve them, and very few chances to learn from their mistakes. Managers will need to deal with complexity and with these changes. Also they need to develop their new 
capabilities to be able to create an effective learning process in complex dynamic systems to overcome the different barriers to learning which are created by complex dynamics systems, and thus to get a significant insight into their simulated problems. This can be done by allowing the managers to develop new ways of thinking that allows deeply understanding of the simulation process, the relations between the variable, and the consequences of their experiments more effectively.

Lots of philosophers, scientist and managers have been calling for a fundamental new ways of thinking that improve the ability to see the world as a complex system. As Sterman [1] stated, "a system in which we understand that you can't just do one thing and that everything else is connected to everything else”. He argued that it's crucial to develop new ways of system thinking. He states that "if people had a holistic worldview, they would then act in consonance with the long term best interest of the system as a whole, identify the high leverage points in systems, and avoid policy resistance”.

This can be done by using new simulation methods that increase the capabilities of managers to experiment their decisions more effectively by using system dynamics simulation tools.

\section{Systems thinking}

The systems thinking concept was produced as the world of systems found that there is a need to shift from the more linear, analytic way of thinking that many of us are used to, to a non linear, dynamic and holistic thinking.

Moving to new the Paradigm in analysing complex problems enables the managers and the analyst to understand dynamic relationships and complexity that influence the behaviour of a system as shown below

\subsection{Features of systems thinking}

\subsubsection{Dynamic and non-linear thinking}

As discussed before, the static thinking assumes that causality runs only one way and any system's factors are independent which is quite primitive. Dynamic thinking offers effective alternatives to see and understand systems or problems. This creative thinking allows viewing the world with ongoing, interdependent relations, dynamic process. Each of the causes in the dynamic thinking is linked in a circular process to both the effect and to each of other causes. These circular processes are the feedback loops which enable us to better understand what is going on in the system; these circular loops represent a non-linear and dynamic thinking (Richmond [2]).

Taking into consideration this type of thinking, the analyst or the manager can understand the problem in a better way as the feedback process inside a firm clarifies the dynamic relations inside a firm, analysing the causes and effects and their interconnection and allows for observing the behaviour over time.

For example, if a firm decreases its product's price, this decision has an effect on the sales as it increases the sales, but on the other hand the firm's profits will 
be less than usual, which affects the firm's pricing policy and push the firm to increase the prices.

\subsubsection{Holistic thinking}

Holistic thinking is one of the most significant features of systems thinking as it allows us to see the "Big Picture". So instead of examining each part of the system, the whole system is examined. Whatever the problem we are experiencing and searching for its source, we must always widen our focus to include that bigger system. Dealing with the wholes rather than parts is a very effective idea in system analysis. Each part or department in a firm is not isolated from other department, so trying to solve a problem in one process; we must first look the whole firm and the interconnections inside it to understand the nature and the reasons for such problem.

This research illustrated how systems thinking tools provides managers and analysts with a creative holism

\subsubsection{Systemic thinking}

In recent years, systems thinking has provided new effective methods for tackling issues in a systemic than a reductionist way. Systems thinking allow us to look for various patterns of behaviour, to seek underlying systemic interrelationships which are responsible for these types of behaviour and events.

A recent study by Bartlett [3] defines systemic thinking as a technique that provides a deeper insight into complex situation very quickly. It stated that Systemic thinking "combines analytical thinking (breaking things apart) with synthetical thinking (putting things together)" as the next figure shows.

This provides more effective holistic and systemic analysis of the system.

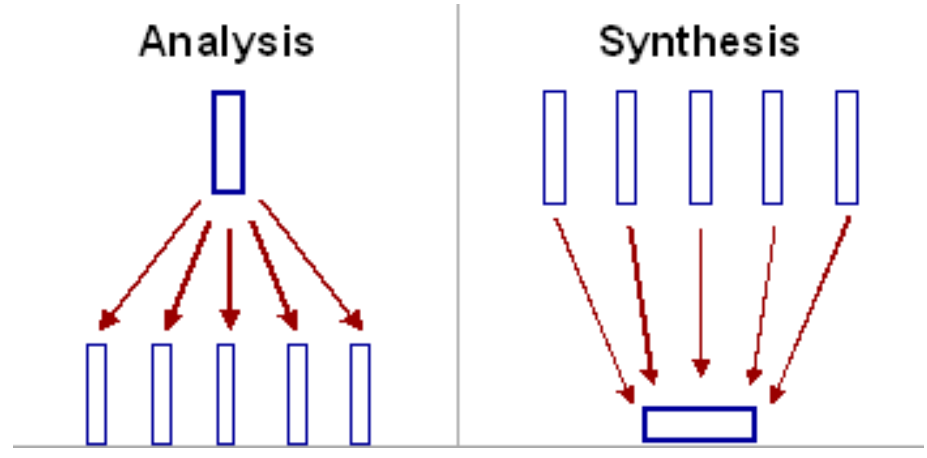

Figure 1: $\quad$ Analysis and synthesis (Bartlett [3]).

\subsection{Systems thinking approaches}

Systems thinking approaches can be divided into two categories, hard systems and soft system. Hard systems thinking is a generic title defined by Checkland [4] to various types of systems approaches for solving problems in the real world. The approaches related to hard systems are operational research, system 
analysis and system dynamics. It is a useful way of thinking which gives the managers and the decision makers the ability to optimize and control the system's performance in pursuit of clearly identified goals.

Another approach is introduced to help to decide what purpose the organisation should pursue which is soft systems methodology and cybernetics.

In this paper, we will introduce the System Dynamics methodology.

\subsubsection{System Dynamics}

System Dynamics methodology is considered an important approach which has a sophisticated tools such as Virtual world (formal models, Microworld, management flight simulators, computer simulation) in which decision makers and managers can refresh decision making skills, test their scenarios and strategies, and conduct experiments through effective simulation by using system Dynamics simulation tools.

System Dynamics is a computer-aided method for analysing, framing, understanding, solving problems in complex real world systems. Jay W. Forrester's work at the Massachusetts Institute of Technology was the major move towards developing this field to extend the range of applied system thinking to more strategic problems in complex systems.

System Dynamics approach uses a perspective based on information feedback and delays to understand the dynamic behaviour of complex physical, biological, and social systems. It also helps the decision maker untangle the complexity of the connections between various policy variables by providing a new language and set of tools to describe. It even models the cause and effect relationships among these variables $[1,7,8]$.

Furthermore, System Dynamics method enables the decision makers or the modeller via its tools in any system to identify the underlying structure of their system or issue and how this structure determines the system's behaviour as shown in the diagram in figure 2, The left arrow symbolizes the relationship while the right arrow indicates the deeper understanding that happens from analysing a system structure. System Dynamics can also be used to study the changes in one part of a system in order to observe its affect on the behaviour of the system as a whole (Martin [5]). Sterman [1] gives an insight that the real value of an SD model should be to eliminate problems by changing the underlying structure of the system rather than anticipating and reacting to the environment. This allows the model to interact with the environment and gives/alerts feedback for structure changes. This is what the term (Dynamics) refers to, i.e. the changes in the system's variables while interacting which stimulate changes over time.

Lyneis [6] stresses the importance of System Dynamic simulation and its power to forecast a market demand for instance and compares this with a statistical forecast. He mentions that an SD Model provides more reliable forecasts than the statistical (non-structural) models and tries to understand the underlying structure that created the data stream. 


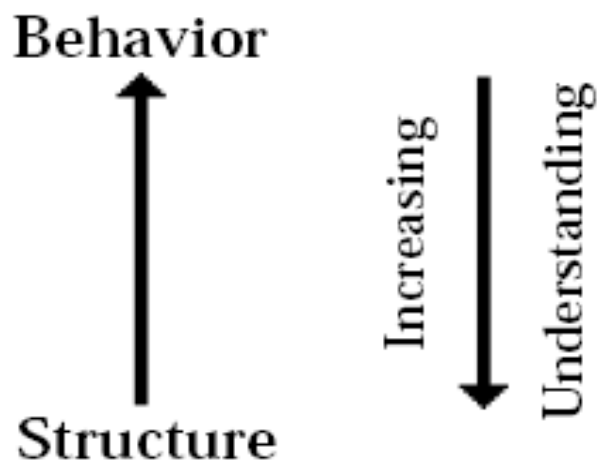

Figure 2: The link between structure and behaviour.

There is lots of user friendly System Dynamics software available now that allows conversion of causal loop diagram and stock and flows diagram into sophisticated computer simulation of the problems or issues being investigated. Examples of these different kinds of software are DYNAMO, STELLA, ITHINK, VENSIM, and POWERSIM (the latest is being used in my study).

Initial values are identified for the stocks, variables values are also identified for the relationships, and the structural relationships are determined between the variables using constants, graphical relationships, and mathematical functions where appropriate. The computer simulation software also facilitates the creation of Microworlds (or management flight simulators) which are kinds of System dynamics Simulators $[7,8]$ as shown in the case study.

\section{Case study}

In this section, a simplified generic system dynamics model of a small firm has been built. This model can be used to analyze the firm which sells product or services to its customers and control the money owed to the firm by its customers which is shown in its accounts as an asset called account receivables.

Figure 3 shows the account receivable structure which represents the total monies owed the firm by its customers on credit sales made to them. It depends mainly on the credit policy given to the customer to encourage them to increase their purchases from the firm.

Collecting the sales revenue from the customers, new loans, and the owners investment are the main income to the firm which increases the cash as shown in figure 4 . The cash is the most liquid asset in the firm that is always ready to be used to pay bills, pay supplier, repayment of bank loans, and much more expected and unexpected outlays. 


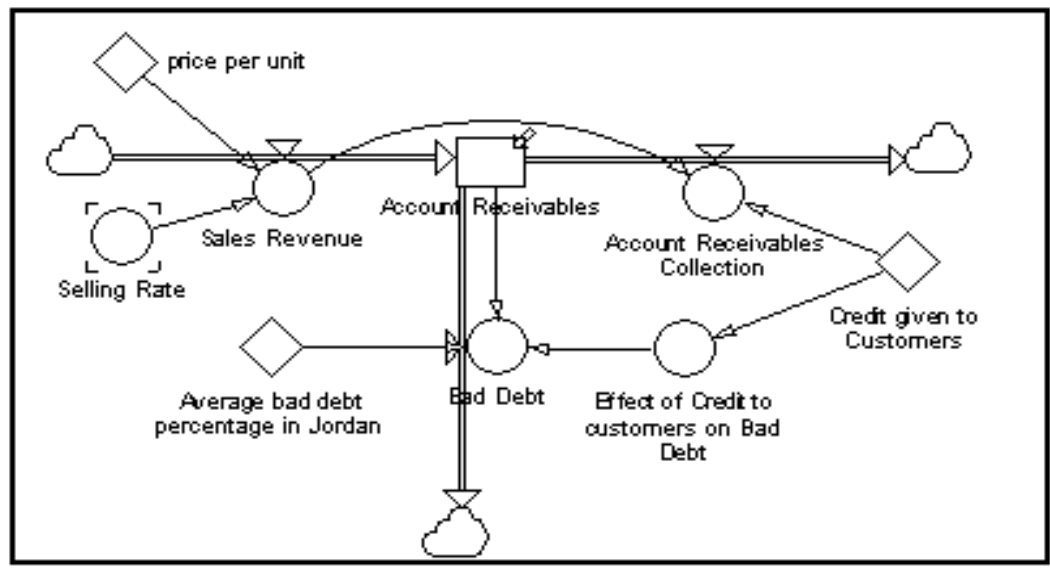

Figure 3: Account receivables structure.

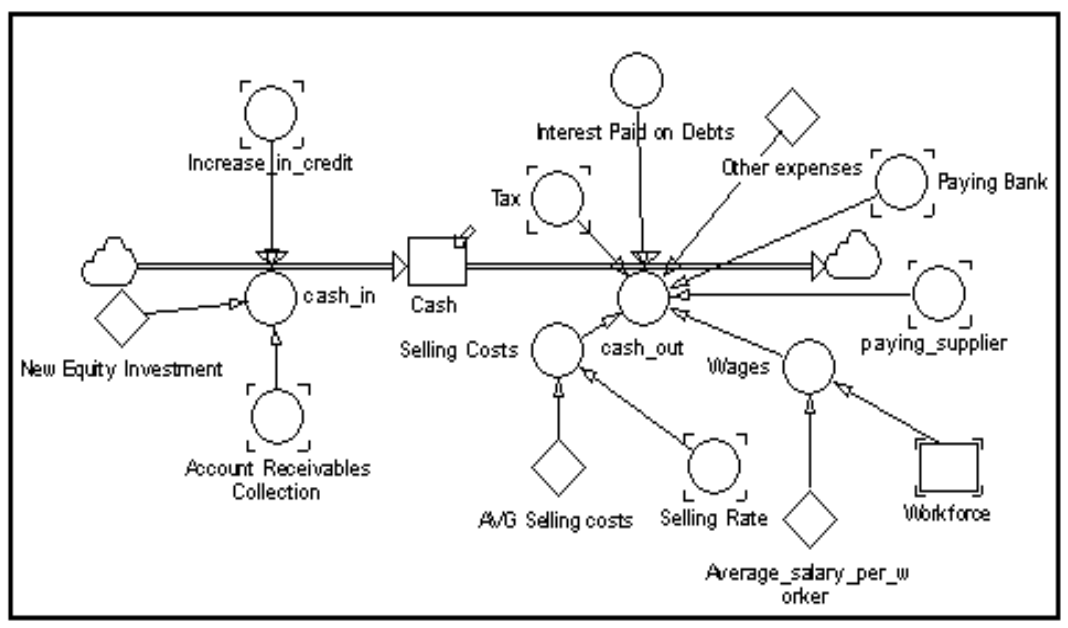

Figure 4: $\quad$ The cash structure.

\subsection{The Simulator}

The Simulator's friendly interface is designed to enable a financial manager to analyse a firm more effectively. The manager inputs the initial data to the simulator, interacts with various windows according to what variables or decisions he is analysing and selects the reports or graphs he needs. The manager is able to change his decisions about how long should the firm provide credit facilities to their customer, and observe the outcome feedback and result in another window. In this paper, we present only the account receivables window which has the system dynamics inside. 
The manager has the opportunity to change the credit term allowed by the firm to its customers. This shows the analyst the behaviour of the firm if any changes in the credit terms happened and its effect on the monthly repayment of loan, interest rate, and the line of credit approved by the bank.

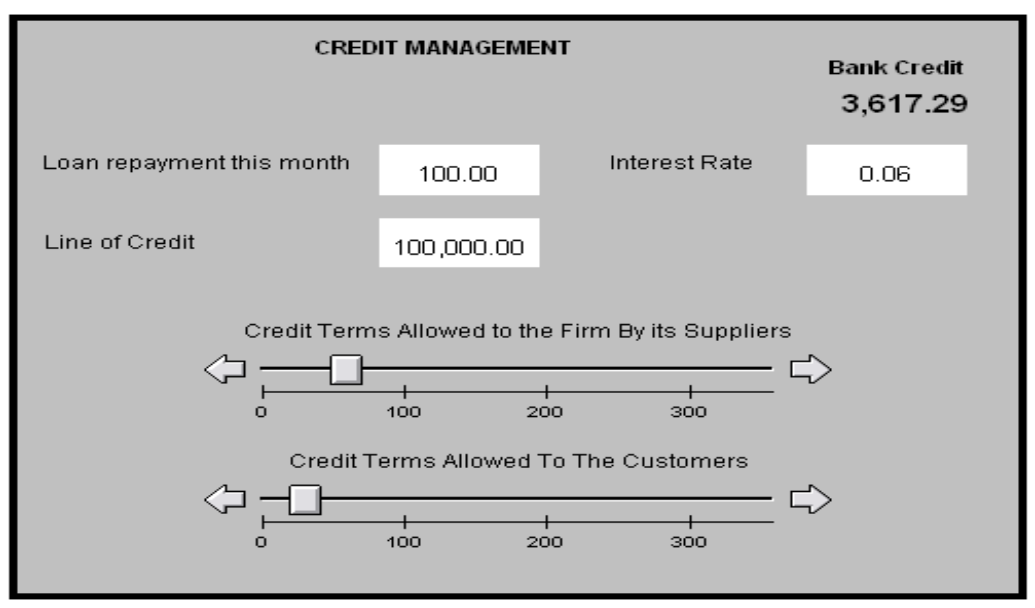

Figure 5: Credit Management Window.

\subsubsection{Running the Simulator}

If the firm is collecting its revenue from its customer immediately without allowing them to delay their payment, then it won't need to withdraw cash from its bank credit as shown in the manager first scenario.

This scenario increase the sales orders because of the open credit policies, as assumed in this scenario, the firm encourages the customers to buy its product with a credit offer to pay after 60 days. As a consequence of this decision, the cash will rapidly decrease and the firm will need to withdraw from its credit line as shown in figure 6 to cover the cash shortage.

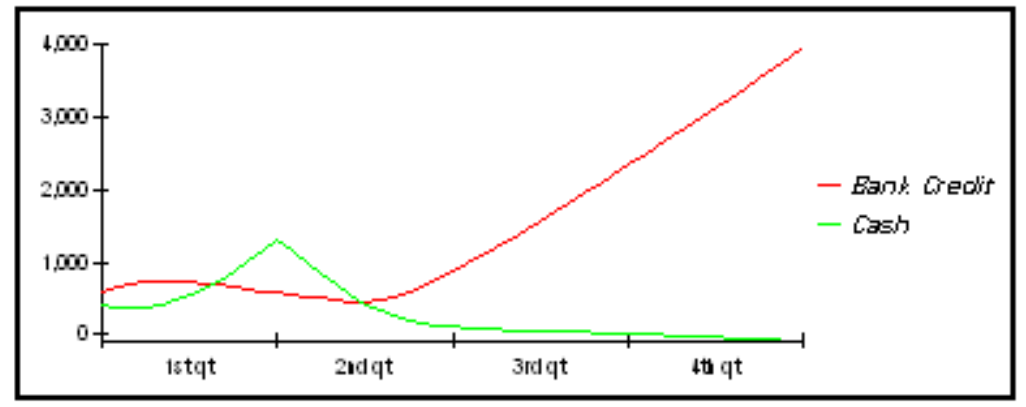

Figure 6: The behaviour of Cash and Bank Credit. 
Analyzing the performance of the firm from the financial manager point of view does not concern only with these graphs, but most likely is to analyse the financial ratios and the changes that might happen to these ratio when simulating the scenario. Two ratios were used here to demonstrate these changes.

\begin{tabular}{|c|c|c|}
\hline Time & Current Ratio & Avg Collection period \\
\hline $01 / 01 / 2007$ & 1.43 & 7.75 \\
\hline $01 / 02 / 2007$ & 0.72 & 6.89 \\
\hline $01 / 03 / 2007$ & 0.80 & 4.68 \\
\hline $01 / 04 / 2007$ & 0.95 & 3.76 \\
\hline $01 / 05 / 2007$ & 1.21 & 3.73 \\
\hline $01 / 06 / 2007$ & 1.47 & 3.81 \\
\hline $01 / 07 / 2007$ & 1.71 & 3.71 \\
\hline $01 / 08 / 2007$ & 1.88 & 3.55 \\
\hline $01 / 09 / 2007$ & 2.08 & 3.43 \\
\hline $01 / 10 / 2007$ & 2.23 & 3.37 \\
\hline $01 / 11 / 2007$ & 2.40 & 3.29 \\
\hline $01 / 12 / 2007$ & 2.48 & 3.22 \\
\hline $01 / 01 / 2008$ & 2.59 & 3.15 \\
\hline & & \\
\hline
\end{tabular}

Figure 7: Current and average collection period ratios before simulating the scenario.

Examples of some of two main ratios that have significantly changed when simulating the scenario are shown in figure 7 which shows the historical changes during one year when the days allowed to customer's payment $=0$, while figure 8 depicts the changes that happened to these ratios when the firm expand its credit policy. As assumed before, the firm offer the customers 60 days to pay for their purchases.

\begin{tabular}{|c|c|c|}
\hline Time & Current Ratio & Avg Collection period \\
\hline $01 / 01 / 2007$ & 1.43 & 7.75 \\
\hline $01 / 02 / 2007$ & 0.71 & 19.83 \\
\hline $01 / 03 / 2007$ & 0.80 & 22.56 \\
\hline $01 / 04 / 2007$ & 0.87 & 28.60 \\
\hline $01 / 05 / 2007$ & 0.95 & 40.63 \\
\hline $01 / 06 / 2007$ & 0.99 & 52.97 \\
\hline $01 / 07 / 2007$ & 1.00 & 61.33 \\
\hline $01 / 08 / 2007$ & 0.98 & 67.32 \\
\hline $01 / 09 / 2007$ & 0.96 & 73.31 \\
\hline $01 / 10 / 2007$ & 0.93 & 79.63 \\
\hline $01 / 11 / 2007$ & 0.90 & 84.88 \\
\hline $01 / 12 / 2007$ & 0.87 & 89.52 \\
\hline $01 / 01 / 2008$ & 0.83 & 93.51 \\
\hline & & \\
\hline
\end{tabular}

Figure 8: Current and average collection period ratios with simulated scenario. 
As a consequence of expanding the credit policy given to its customers, the risk of collecting cash increased in the firm which is a normal result of the new policy. The average collection period increased from 3 days to 93 days at the end of the year which means that the firm will collects its sales revenue within 93 days, which in turn decreases the cash balance in the firm, and reduces the current ratio which measures the firm's ability to meet its financial obligations.

Current ratios of 2.59 as on $1 / 1 / 2008$ before simulating the scenario is occasionally cited as good ratio, while a current ratio of 0.83 as on 1/1/2008 after simulating the new policy would be normally considered unacceptable.

On the other hand, implementing this scenario might be useful as it encourages the customers to place more orders and increases the sales in the firm.

The analyst was able to test and observe whatever ratios and scenarios he found vital to analyse the firm's performance from different aspects with many times to try.

\section{Conclusion}

It is clear now that Systems Thinking tools especially System Dynamics are better than others because it can easily deal with non-linearities and time which are not considered by a static analysis.

By applying System Dynamics, one can enhance the usefulness of the model to address and analyse problems in a complex situations and provide more significant, rational and pertinent policy recommendations.

In summary, the process is to "observe and identify problematic behaviour of a system over time and to create a valid diagrammatic representation of the system, capable of reproducing by computer simulation the existing system behaviour and facilitating the design of improved system behaviour. Existing business simulators are designed to allow the users to play a realistic role in management decision making. Users can make decisions and receive outcome feedback about their performance, by rehearsing strategies and observing results, the managers in the case study were able to discover how to make better decisions and hence improve their performance and reduce the risk of losing money and thus increase the firm's financial performance as illustrated.

\section{References}

[1] Sterman, J., Business Dynamics: System Thinking and modelling for a complex world, USA, 2000

[2] Richmond, B., Systems thinking: critical thinking skills for the 1990's and beyond. System dynamics review, 9(2), pp. 113-133, 1993

[3] Bartlett, G., Systemic thinking: a simple thinking technique for gaining systemic focus. In the international conference on thinking, breakthroughs, USA, 2001

[4] Checkland, P., Systems Thinking: Systems Practice, John Wiley \& Sons, 1981 
[5] Martin, L., Mistakes and Misunderstandings: System Dynamics in Education Project. System Dynamics Group, Sloan School of Management, Massachusetts Institute of Technology, 1997

[6] Lyneis, J., System dynamics for market forecasting and structural analysis. System Dynamics Review, 16(1), pp. 3-25, 2000

[7] Gonzalez, C., The use of Microworlds to study dynamic decision making. Dynamic Decision Making Laboratory, Carnegie Mellon University, USA, 2004

[8] Langley, P. \& Morecroft, J., Performance and learning in a simulation of oil industry dynamics. European Journal of Operational Research, (155), pp. 715-732, 2004 